\title{
A Comparative Analysis of Hybrid Approach for Gene Cancer Classification using Genetic Algorithm and FFBNN with Classifiers ANFIS and Fuzzy NN
}

\author{
Vaishali P Khobragade ${ }^{1}$, Dr.A.Vinayababu ${ }^{2}$ \\ ${ }^{1}$ Jyothishmathi Institute of Technology \& Science, CSE Department, JNTU Hyderabad, Karimnagar, Andhra \\ Pradesh 505001, India \\ ${ }^{2}$ Principal JNTUH College of Engineering, JNT University, Hyderabad, Andhra Pradesh 500085, India
}

\begin{abstract}
With the advent of microarray technology Many algorithms and techniques were developed for the microarray gene classification process. These developed techniques accomplish microarray gene classification process with the aid of three basic phases namely, dimensionality reduction, feature selection, and gene classification. In our work, microarray gene classification by GA with FFBNN was proposed for precise classification of genes to their corresponding gene types. But, it is not sure that the GA and FFBNN will perform their operations properly in gene classification process. Thus, analysis is necessary for the techniques that are utilized in the gene classification process. Hence, in this study, we present a comparative analysis of familiar methods that are utilized in the microarray gene classification process. The performances of wellknown methods such as GA (Genetic Algorithm) are analyzed with the AI techniques namely, FFBNN, Fuzzy $N N$, and ANFIS. The methods that are used for microarray gene classification process are GA with FFBNN, GA with Fuzzy NN, GA with ANFIS.The microarray gene expression dataset dimension is reduced by GA, and then the selected features from the GA method is used to train the networks such as FFBNN, Fuzzy NN and ANFIS and these well trained networks classifies the microarray gene values into their corresponding cancer class types. The performances of the classification methods are evaluated by the performance measures such as accuracy, specificity, and sensitivity. Moreover, the classification performance of each method is compared with the other methods to validate the high score performance in microarray gene classification.
\end{abstract}

Keywords - Microarray gene expression, Classification, Dimensionality Reduction, Feature Selection, Genetic Algorithm (GA), Feed Forward Back propagation Neural Network (FFBNN), Adaptive Neuro-Fuzzy Inference System (ANFIS), Fuzzy neural Network (FNN).

\section{INTRODUCTION}

With the aid of deoxyribonucleic acid (DNA) microarray technology, it is possible to determine the expression levels of vast number of different genes simultaneously [9]. Microarray techniques also play an imperative role in personalized medicine for the reason that they can be used to find out the individual's unique genetic vulnerability to treat the diseases [1]. A standard microarray dataset comprises the expression levels of large number of genes in a number of experimental samples or conditions [10]. The expression data is represented in a matrix form, where the rows indicate genes and the columns indicate samples and this form of matrix is called as gene expression matrix [11]. For disease analysis especially for cancer diagnosis, the gene expression data is often employed [8]. Gene expression data from DNA microarray are represented by several variables (genes) with only a small number of observations (experiments) [7] [17]. Prediction, classification, and clustering methods are utilized for analysis and understanding of the data [2]. One significant application of gene expression microarray data is the classification of biological samples or prophecy of clinical and other outcomes [3]. Microarray technology categorize the tissue samples by means of their gene expression profiles as one of the several types (or subtypes) of cancer. The gene expression profiles evaluated by microarray technology have offered a precise, consistent and objective cancer classification than the standard histopathological experiments. The DNA microarray data for cancer classification comprises huge number of genes (dimensions) than the number of samples or feature vectors [4] [18]. The gene expression variation of different tumor types is evaluated by using the genome-wide expression data obtained from the cancer tissues, which further provides hints for cancer classification of individual samples. Determining biological insights from the original amount of data on gene expression patterns is the major challenging tasks in microarray studies [12]. A robust model is indispensable for predicting the class membership of data, creating an exact label on training data, and predicting the label for any anonymous data correctly in order to achieve a high classification accuracy [8]. Classification analysis of microarray gene expression data has been carried out extensively to determine the biological features as well as to differentiate intimately related cell types that 
normally appear in the diagnosis of cancer [13]. Some of the classification techniques for gene expression data analysis are decision tree, k-nearest neighbor classifier (KNN), support vector machine (SVM), neural network, etc. Normally, the techniques used for the classification of microarray gene expression data are divided into two groups: one is based on clustering and the other is based on machine-learning approach [14]. There are many techniques developed for the microarray gene classification. In classification, the genes in the microarray dataset are classified into their corresponding class types. Normally, all microarray gene classification techniques perform three basic steps during the classification process, they are: dimensionality reduction, feature selection, and gene classification. In our prior work, microarray gene classification was performed by GA with FFBNN for precise classification of genes to their respective gene types. However, it is uncertain that the GA and FFBNN will perform their operations properly in gene classification process. Thus, an analysis is essential for the techniques that are utilized in the gene classification process. Hence, here we proposed a comparative analysis of well known methods that are used in the microarray gene classification process. The performances of well-known methods such as GA (Genetic Algorithm) are analyzed with the AI techniques namely, FFBNN, Fuzzy NN, and ANFIS. The methods that are employed for microarray gene classification process are GA with FFBNN, GA with Fuzzy NN, GA with ANFIS. All these methods separately perform the aforementioned three basic steps. The performances of the classification methods are evaluated by the performance measures such as accuracy, specificity, and sensitivity. The rest of the paper is organized as follows: Section 2 reviews the recent related works of the microarray gene classification process. The well known classification method such as GA and AI techniques FFBNN, Fuzzy NN and ANFIS are explained in Section 3. The experimental result and conclusion of this paper are given in Section 4 and 5, respectively.

\section{RELATED WORK}

Plenty of researches have been carried out for the successful microarray gene cancer classification. A handful of recent works available in the literature are reviewed in this section.Ahmad M. Sarhan [20] has introduced an Artificial Neural Network (ANN) and Discrete Cosine Transform (DCT) based system for the identification of stomach cancer. Here, DCT has been applied to extract the classification features from the stomach microarrays. Subsequently, the features extracted from the DCT coefficients have been applied to an ANN for the classification in order to find whether the microarray contains tumor or non-tumor. Here, microarray images have been taken from the database called Stanford Medical Database (SMD), which is one of the famous microarray databases. From the simulation results, it has been found that the proposed system has achieved a very high success rate.Bharathi et al. [21] have aimed to identify the minimum set of genes that can provide an exact classification of cancer from microarray data with the aid of supervised machine learning algorithms. The significance of determining the smallest gene subset has three advantages: (i) the computational burden and noise occurred from irrelevant genes have been diminished substantially, (ii) the gene expression tests have been simplified to comprise only a very less number of genes instead of thousands of genes, so the expenditure for cancer testing has been reduced considerably, and (iii) further analysis has been made into the possible biological relationship between these small number of genes and cancer development and treatment. The proposed method comprises two steps. In the first stage, a 2 way Analysis of Variance (ANOVA) ranking approach has been employed to select some relevant genes. While in the second stage, a good classifier called Support Vector Machines has been applied to analyze the classification potency of all simple combinations of those relevant genes. Finally, the proposed method has achieved a very high precision with only two genes. Gene expression data gathered from DNA microarray are characterized by several variables (genes) with only a little number of observations i.e., experiments. Bo Li et al. [22] have presented a manifold learning technique to map the gene expression data to a low dimensional space, and then to analyze the basic structure of the features in order to categorize the microarray data more precisely. The proposed algorithm has projected the gene expression data into a subspace with high intra-class compactness and inter-class separability. Experiments conducted on six DNA microarray datasets have proved that the proposed method was efficacious for discriminant feature extraction as well as gene expression data classification. It has been found that evaluating microarray data using manifold learning technique is a valuable effort and there should be much room for the application of manifold learning to bioinformatics because of its performance.Xiaosheng Wang et al. [23] have examined the properties of one feature selection scheme proposed in their prior work, which was the simplification of the feature selection technique based on the depended amount of attribute in rough sets. Here, the feature selection technique has been compared with the conventional methods in terms of depended degree, chi-square, information gain, Relief-F and symmetric uncertainty, and its properties have been evaluated by a series of classification experiments. The experimental results have exposed that the proposed approach was better than the canonical depended degree of attribute based technique in effectiveness and applicability. Moreover, the approach has been compared with the other four widely used techniques. It has been found that the proposed approach can disclose the inherent classification difficulty with respect to diverse gene expression datasets, representing the intrinsic biology of specific cancers. Mallika et al. [24] have introduced a technique 
for enhancing the cancer classification performance with a small number of microarray gene expression data. Here, individual gene ranking and gene subset ranking have been carried out. Also, the same classifier has been employed for both selection and classification purposes. The proposed technique has been tested using three eminent cancer gene expression datasets namely Lymphoma, Liver, and Leukaemia datasets. Three diverse classifiers such as Support Vector Machines-One Against All (SVM-OAA), K Nearest Neighbour (KNN) and Linear Discriminant Analysis (LDA) have been evaluated and the results have revealed that the performance of SVM-OAA classifier was better and has provided a satisfactory results on all the three datasets than the other two classifiers. Chhanda Ray [25] has proposed an algorithm to inspect the DNA microarray gene expression patterns robustly for large amount of DNA microarray data. Graphical representation has been presented for the experimental results of DNA microarray gene pattern analysis for improved visibility and understanding. An eight-directional chain code sequence has been employed to define the shape of each graph related to a DNA microarray gene expression pattern. Based on the variations of DNA microarray gene expression patterns of the same organism by concurrently monitoring the behaviors of thousands of genes, the cancer development has been detected. Moreover, the classification of cancer genes has been carried out on the basis of distribution probability of codes of the eight-directional chain code sequences indicating DNA microarray gene expression patterns. Finally, an experimental result has been presented. DNA microarrays allow the biologist to evaluate the performance of thousands of genes concurrently on a small chip. These microarrays produce giant number of data and new techniques are necessary to evaluate them. Seeja et al. [26] have proposed an SVM based classification technique. The gene expression data recorded on DNA microarrays has been classified by using this proposed technique. The proposed technique has been tested by using benchmark datasets and it has been found that the technique was faster than neural network and the classification performance was also high compared to neural network.

\section{MICROARRAY GENE CANCER CLASSIFICATION}

The microarray gene classification technique involves three major steps namely (i) Dimensionality reduction, (ii) Feature selection, and (iii) Gene classification. The GA technique performs the dimensionality reduction process for obtaining the dataset with small size. The features like Standard Deviation, Probability of GA-indexed gene, and new statistical features are extracted from the dimensionality reduced dataset. After that, the gene classification is carried out by using the features extracted during the feature extraction process. The AI techniques such as FFBNN, Fuzzy NN, and ANFIS perform the gene classification process. The basic microarray gene classification process is explained in the following subsections.

\section{DIMENSIONALITY REDUCTION}

Initially, the dimensionality reduction process is carried out on the microarray cancer gene dataset for diminishing the complexity in the gene classification. This process is performed because the dataset size is high dimensional, which increases the processing time and does not produce accurate result for the classification process. Let, $M_{i j} ; 1 \leq i \leq S, 1 \leq j \leq G$ be the microarray cancer gene data, where, $S$ indicates the number of samples and $G$ indicates the number of genes. Dataset $M_{i j}$ contains $N$ number of cancer class types, which is represented as $D_{c}=\left\{l_{1}, l_{2}, \cdots l_{N}\right\}$. The gene dataset can be represented as,

$$
M_{i j}=\left[\begin{array}{cccc}
g_{(1,1)} & g_{(1,2)} & \cdots & g_{(1, G)} \\
g_{(2,1)} & g_{(2,2)} & \cdots & g_{(2, G)} \\
\vdots & \vdots & \vdots & \vdots \\
g_{(S, 1)} & g_{(S, 2)} & \cdots & g_{(S, G)}
\end{array}\right]
$$

Each row and column of the gene expression dataset index values are represented as,

$$
R_{i}=\left\{r_{1}, r_{2}, \cdots r_{G}\right\}, C_{j}=\left\{o_{1}, o_{2}, \cdots o_{S}\right\}
$$

\section{DIMENSIONALITY REDUCTION BY GA}

The dimensionality reduction by GA process is briefly explained in the prior work. Initially in GA, the

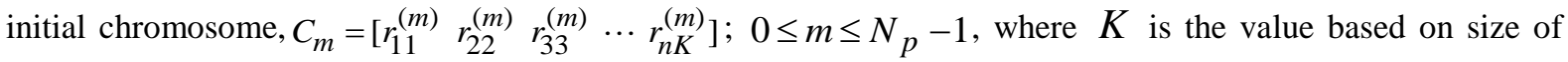
the chromosome and $n$ represents the genes row index value in $M_{i j}$ where $n \in r_{G}$. The fitness function is carried out to choose the best chromosomes among the generated chromosomes. The fitness function is given as, 


$$
f 1=\frac{S^{\left(C_{m}^{(s)}\right)} * S^{\left(l_{1}\right)} * S^{\left(l_{2}\right)} * \ldots S^{\left(l_{N}\right)}}{E^{\left(C_{m}\right)} * T^{\left(C_{m}\right)} * t_{1}}
$$

where, $S^{\left(C_{m}^{(s)}\right)}$ is the standard deviation of the chromosome $C_{m}^{(s)}$ and $S^{\left(l_{1}\right)}, S^{\left(l_{2}\right)}, \cdots S^{\left(l_{N}\right)}$ are also the standard deviations of the genes cancer class types. All the generated chromosomes gene values are given to the networks such as FFBNN, ANFIS and Fuzzy ANN to obtain the error $\left(E^{\left(C_{m}\right)}\right)$ and time $\left(T^{\left(C_{m}\right)}\right)$ parameters of the chromosome $C_{m} . E^{\left(C_{m}\right)}$ is the error produced when the networks are trained by the chromosome $C_{m}$. Time parameter $T^{\left(C_{m}\right)}$ represents the time taken by the networks to train the $C_{m}$, and $t_{1}$ is the defined threshold value. The best $N_{p} / 2$ chromosomes containing minimum fitness values are selected. The selected chromosomes are involved in the crossover and mutation operations with the single point crossover at crossover rate $C_{R}$ and mutation rate $M_{R}$, respectively. This process is repeated until it reaches the utmost number of iterations $I$. Once it reaches $I$, the $N_{p} / 2$ chromosomes having minimum fitness value are selected. The dimensionality reduced dataset from GA is represented as $P_{u v}$.

\section{FEATURE SELECTION}

The features are selected from the dimensionality reduced datasets $P_{u v}$ and $P_{u v}^{\prime}$. The features like Standard Deviation, Probability of GA-indexed gene, and new statistical features are selected from the dimensionality reduced dataset. The features that are selected from the dataset $P_{u v}$ are briefly explained in the previous work. From this dataset, the extracted features are $F^{(d)}, F^{(c)}, F^{(A)}, F^{(D)}, F^{(p)}$ and $F^{(s s)}$. Also, the similar features are extracted from the dataset $P_{u v}^{\prime}$, which is represented as $F^{(d)^{\prime}}, F^{(c)^{\prime}}, F^{(A)^{\prime}}$, $F^{(D)^{\prime}}, F^{(p)^{\prime}}$ and $F^{(s s)^{\prime}}$.

\section{GENE CLASSIFICATION}

Using the SD, Probability of GA-indexed gene, and new statistical features determined in the previous phase, the gene classification process is carried out. To perform the classification process, here we utilized three AI techniques such as Feed Forward Back Propagation Neural Network (FFBNN), ANFIS, and Fuzzy NN. Each technique is trained and tested with the features that are obtained from the dataset $P_{u v}$ and $P_{u v}^{\prime}$, individually.

\section{CLASSIFICATION USING FFBNN}

Classification by FFBNN using the features from $P_{u v}$ is already explained in our previous work. In this classification process, the FFBNN is designed with six input neurons, $H_{d}$ hidden layers, and one output layer. The FFBNN training process is performed with the bias and activation functions of input and output layers, respectively. After that, the network learning error rate is calculated and the error gets minimized by allocating weights to the hidden layer and output layer neurons via back propagation algorithm. Testing process is done for the column gene values in the dimensionality reduced dataset $P_{u v}$. The well trained FFBNN classifies the column gene values into any one of the cancer class types by using the extracted features. The same FFBNN training and testing process is performed with the features from $P_{u v}^{\prime}$. 


\section{CLASSIFICATION USING ANFIS}

ANFIS architecture comprises five layers of nodes. Out of the five layers, the first and fourth layers are the adaptive nodes while the second, third and fifth layers are the fixed nodes. ANFIS has a five layered feedforward neural network structure, which is shown in figure 2. Both artificial neural network (ANN) and fuzzy logic (FL) are employed in the ANFIS architecture. In both ANN and FL, the input is passed to the input layer (as input membership function) and the output is obtained from the output layer (as output membership functions). Here, a Sugeno type model is used that makes use of if-then- rules to produce an output for each rule. Rule outputs consist of linear combination of the input variables plus a constant term, and the final output is the weighted average of each rule's output. Sugeno type if-then-rules are defined for both dimensionality reduced datasets by the input values i.e., extracted features. Each layer in the ANFIS system is processed with the input values that are briefly explained in [28]. The ANFIS system is well trained by the features from $P_{u v}$ and the same training process is performed in ANFIS with the features from $P_{u v}^{\prime}$. Testing process is performed in ANFIS with the one column of gene values in the dataset and the remaining columns gene values are utilized in the training process.

Figure 2: Basic ANFIS Architecture

\section{CLASSIFICATION USING FUZZY NN}

A fuzzy neural classifier (NFC) [29] is applied on the detected patterns to carry out the sleep-waking state-stage classification. Each of the five significant patterns is related to two fuzzy concepts, present and absent, with sigmoidal fuzzification functions. The NFC architecture allows us to implement a fuzzy classification system with sigmoidal fuzzification functions at layer 1 including parameters that were trained by the delta rule with the squared error as the objective function. The output value is obtained from the layer 3 . The neuro-fuzzy classifier is applied to our gene classification problem with six inputs and one output class. The neuro-fuzzy classifier is individually trained with both dataset features. The NFC testing process is performed with the column gene values same as that of the ANFIS system.

\section{EXPERIMENTAL RESULT}

The proposed classification technique is implemented in the MATLAB platform version 7.8 and evaluated using the microarray gene expression dataset. The dataset contains number of genes and samples i.e., $675 \times 156$. The high dimensional dataset is subjected to dimensionality reduction using a GA and PSO. The dimensionality reduced dataset from GA and PSO are the dimension of 10x156. Among these 156 samples, 1 to 139 samples are AD class type and 140 to 156 samples are NL class type. Then, the feature selection process is performed over the dimensionality reduced datasets and these selected features are given to the FFBNN, ANFIS, FNN training and testing process. The FFBNN training and testing process is explained in our previous work. Similar to FFBNN, the ANFIS system training process is performed with 156 training samples and testing process with 156 testing samples. But, the FNN training and testing process is totally different from the FFBNN and ANFIS system. In FFBNN and ANFIS, the number of training and testing samples may differ i.e., if 155 samples are utilized for training, then $156^{\text {th }}$ sample is used for testing. But in FNN, the number of training and testing samples taken are same i.e., if we utilize $155^{\text {th }}$ sample for the training process, then the testing process also utilize the same $155^{\text {th }}$ sample. The abovementioned procedure is performed until all samples are involved in both training and testing process. The performance of proposed technique is evaluated by using the statistical measures. The statistical measures [27] are applied to determine the classification performance. The performance analysis has shown that the proposed technique has successfully classified the genes to their 
specified gene types. To analyze the performance of GA and PSO methods, the parameters in GA and PSO values are changed. The parameters of GA such as crossover, chromosome length, and mutation rate are changed as well as the PSO parameters such as population size and chromosome length are also changed.

\section{PERFORMANCE OF GA WITH FFBNN}

From the parameter tuning process, the best and worst case values are selected and the performance of these selected best and worst case values are calculated. The True Positive(TP), True Negative(TN), False Positive(FP), and False Negative(FN) are computed for the GA index values 10,20,30.Using these values the best and worst cases statistical measure values are computed and presented in table 1.

\begin{tabular}{|c|c|c|c|}
\hline $\begin{array}{l}\text { Statistical } \\
\text { Measures }\end{array}$ & $\begin{array}{c}\text { Number of } \\
\text { GA indexed } \\
\text { genes }\end{array}$ & Best Case & Worst Case \\
\hline \multirow{3}{*}{$\begin{array}{c}\text { Sensitivity } \\
(\%)\end{array}$} & 10 & 96.40 & 89.21 \\
\hline & 20 & 99.28 & 89.93 \\
\hline & 30 & 99.28 & 87.77 \\
\hline \multirow{3}{*}{$\operatorname{FPR}(\%)$} & 10 & 52.94 & 58.82 \\
\hline & 20 & 35.29 & 70.59 \\
\hline & 30 & 41.18 & 70.59 \\
\hline \multirow{3}{*}{$\begin{array}{c}\text { Accuracy } \\
(\%)\end{array}$} & 10 & 91.03 & 83.97 \\
\hline & 20 & 95.51 & 83.33 \\
\hline & 30 & 94.87 & 81.41 \\
\hline \multirow{3}{*}{$\begin{array}{c}\text { Specificity } \\
(\%)\end{array}$} & 10 & 47.06 & 41.18 \\
\hline & 20 & 64.71 & 29.41 \\
\hline & 30 & 58.82 & 29.41 \\
\hline \multirow{3}{*}{ PPV (\%) } & 10 & 93.71 & 92.54 \\
\hline & 20 & 95.83 & 91.24 \\
\hline & 30 & 95.17 & 91.04 \\
\hline \multirow{3}{*}{ NPV (\%) } & 10 & 61.54 & 31.82 \\
\hline & 20 & 91.67 & 26.32 \\
\hline & 30 & 90.91 & 22.73 \\
\hline \multirow{3}{*}{ FDR (\%) } & 10 & 6.29 & 7.46 \\
\hline & 20 & 4.17 & 8.76 \\
\hline & 30 & 4.83 & 8.96 \\
\hline \multirow{3}{*}{$\operatorname{MCC}(\%)$} & 10 & 49.00 & 27.20 \\
\hline & 20 & 74.83 & 18.43 \\
\hline & 30 & 70.72 & 15.38 \\
\hline
\end{tabular}

Table 1: Performance of proposed FFBNN best, worst cases .

\section{PERFORMANCE OF GA WITH ANFIS}

In the table 2, the selected best and worst case statistical measure values are presented and compared with the existing SVM classifier, which are shown in table 4. 
A Comparative Analysis of Hybrid Approach for Gene Cancer Classification...

\begin{tabular}{|c|c|c|c|c|}
\hline $\begin{array}{l}\text { Statistical } \\
\text { Measures }\end{array}$ & $\begin{array}{c}\text { Number of } \\
\text { GA indexed } \\
\text { genes }\end{array}$ & Best Case & Worst Case & $\begin{array}{c}\text { SVM } \\
\text { Classifier }\end{array}$ \\
\hline \multirow{3}{*}{$\begin{array}{c}\text { Sensitivity } \\
(\%)\end{array}$} & 10 & 99 & 88 & 91.37 \\
\hline & 20 & 99 & 90 & 94.24 \\
\hline & 30 & 99 & 91 & 94.96 \\
\hline \multirow{3}{*}{ FPR $(\%)$} & 10 & 64.7 & 64.7 & 17.65 \\
\hline & 20 & 41.2 & 70.6 & 23.53 \\
\hline & 30 & 23.5 & 58.8 & 29.41 \\
\hline \multirow{3}{*}{$\begin{array}{c}\text { Accuracy } \\
(\%)\end{array}$} & 10 & 92 & 83 & 90.38 \\
\hline & 20 & 94 & 83 & 92.31 \\
\hline & 30 & 96 & 85 & 92.31 \\
\hline \multirow{3}{*}{$\begin{array}{c}\text { Specificity } \\
(\%)\end{array}$} & 10 & 35 & 35 & 82.35 \\
\hline & 20 & 59 & 29 & 76.47 \\
\hline & 30 & 76 & 41 & 70.59 \\
\hline \multirow{3}{*}{ PPV (\%) } & 10 & 93 & 92 & 97.69 \\
\hline & 20 & 95 & 91 & 97.04 \\
\hline & 30 & 97 & 93 & 96.35 \\
\hline \multirow{3}{*}{ NPV (\%) } & 10 & 86 & 27 & 53.85 \\
\hline & 20 & 83 & 26 & 61.90 \\
\hline & 30 & 87 & 35 & 63.16 \\
\hline \multirow{3}{*}{ FDR (\%) } & 10 & 7 & 8 & 2.31 \\
\hline & 20 & 5 & 9 & 2.96 \\
\hline & 30 & 3 & 7 & 3.65 \\
\hline \multirow{3}{*}{$\operatorname{MCC}(\%)$} & 10 & 52.9 & 21.7 & 61.64 \\
\hline & 20 & 66.4 & 18.9 & 64.56 \\
\hline & 30 & 76.9 & 30.0 & 62.46 \\
\hline
\end{tabular}

Table 2: Performance of proposed ANFIS best, worst cases and existing SVM classifier

\section{PERFORMANCE OF GA WITH FNN}

The GA parameter tuning with FNN system's TP, TN, FP and FN result values are computed with GA index values 10,20,30. Also, the best and worst values are compiuted for the different statistical measures listed in table 3 , the values are compared with the SVM results.

Table 5: GA parameters tuning results of TP, FP, TN and FN values

\begin{tabular}{|c|c|c|c|c|}
\hline \multirow{2}{*}{$\begin{array}{c}\text { Statistical } \\
\text { Measures }\end{array}$} & $\begin{array}{c}\text { Number of } \\
\text { GA indexed } \\
\text { genes }\end{array}$ & Best Case & Worst Case & $\begin{array}{c}\text { Classifier } \\
{[26]}\end{array}$ \\
\hline \multirow{3}{*}{$\begin{array}{c}\text { Sensitivity } \\
(\%)\end{array}$} & 10 & 96 & 91 & 91.37 \\
\cline { 2 - 5 } & 20 & 96 & 86 & 94.24 \\
\hline \multirow{3}{*}{ FPR (\%) } & 30 & 99 & 88 & 94.96 \\
\cline { 2 - 5 } & 10 & 53.3 & 94.1 & 17.65 \\
\cline { 2 - 5 } & 20 & 35.3 & 58.8 & 23.53 \\
\hline \multirow{3}{*}{$\begin{array}{c}\text { Accuracy } \\
(\%)\end{array}$} & 30 & 41.2 & 76.5 & 29.41 \\
\cline { 2 - 5 } & 20 & 92 & 82 & 90.38 \\
\hline \multirow{3}{*}{ Specificity } & 30 & 93 & 81 & 92.31 \\
\cline { 2 - 5 }$(\%)$ & 10 & 47 & 81 & 92.31 \\
\cline { 2 - 5 } & 20 & 65 & 6 & 82.35 \\
\hline \multirow{3}{*}{ PPV (\%) } & 30 & 59 & 24 & 76.47 \\
\cline { 2 - 5 } & 20 & 94 & 89 & 97.59 \\
\hline \multirow{3}{*}{ NPV (\%) } & 30 & 96 & 92 & 97.69 \\
\cline { 2 - 5 } & 10 & 58 & 90 & 96.35 \\
\cline { 2 - 5 } & 20 & 69 & 27 & 53.85 \\
\hline
\end{tabular}


A Comparative Analysis of Hybrid Approach for Gene Cancer Classification...

\begin{tabular}{|c|c|c|c|c|}
\hline \multirow{3}{*}{ FDR (\%) } & 10 & 6 & 11 & 2.31 \\
\cline { 2 - 5 } & 20 & 4 & 8 & 2.96 \\
\cline { 2 - 5 } & 30 & 5 & 10 & 3.65 \\
\hline \multirow{3}{*}{ MCC (\%) } & 10 & 47.8 & 3.3 & 61.64 \\
\cline { 2 - 5 } & 20 & 61.7 & 23.3 & 64.56 \\
\cline { 2 - 5 } & 30 & 70.0 & 10.7 & 62.46 \\
\hline
\end{tabular}

Table 3: Performance of proposed FNN best, worst cases and existing SVM classifier

The statistical performance analysis of the GA method has given 93.7 overall mean accuracy in the best case and in the worst case it has given $82.6 \%$ accuracy as result. To analyze the selection of FFBNN as a classifier with GA, comparision is made with GA using other classifiers like Fuzzy NN and ANFIS. To analyze the performance of the GA methods, these techniques are compared with the existing SVM classifier. This GA method best and worst case statistical measures are compared with the SVM is shown in the figure 3 and 4.

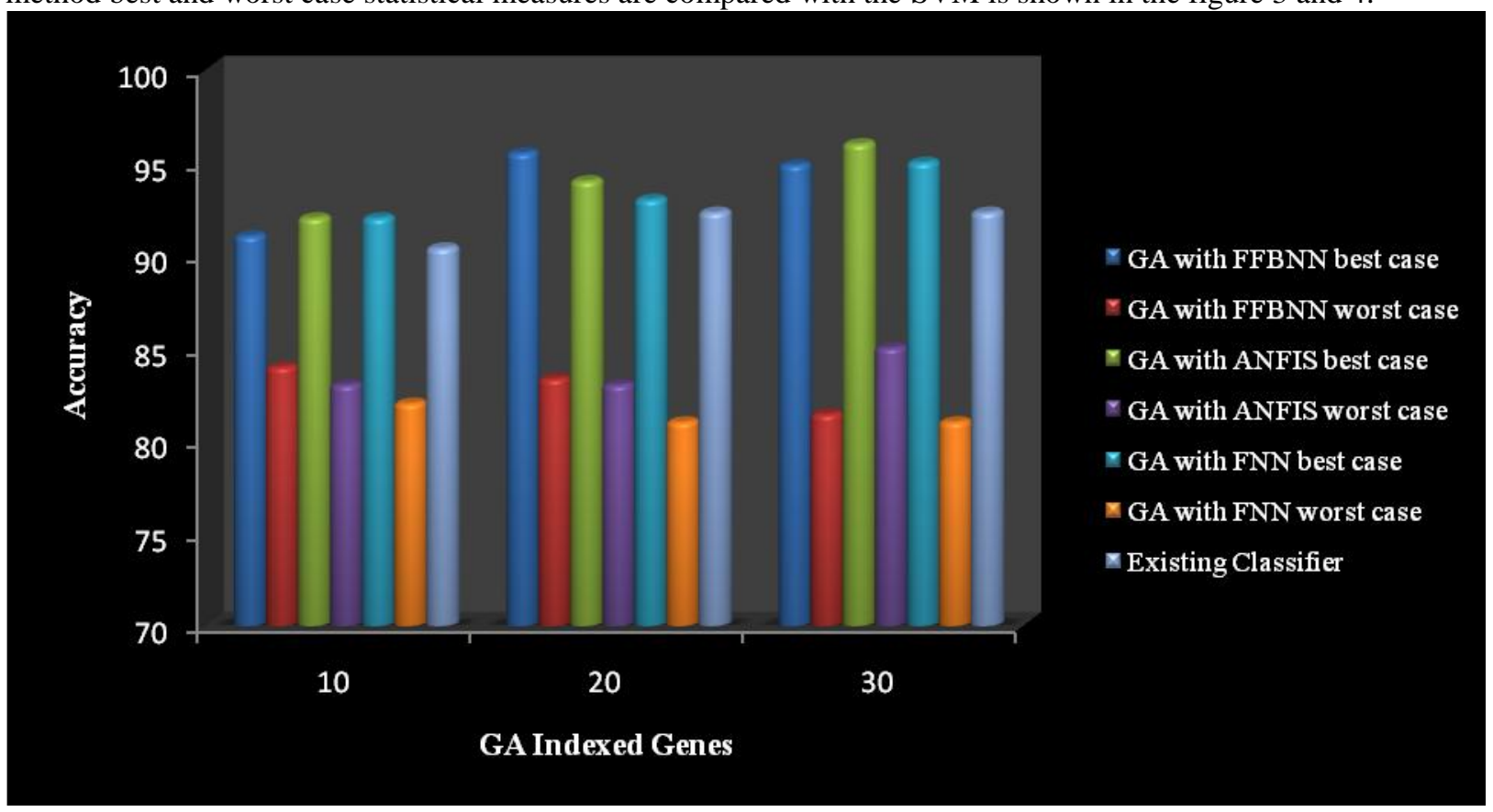

Figure 3: Comparison of GA methods with existing SVM classifier

The comparison graph 3 shows that the existing method has low classification performance than GA in all 10, 20 and $30 \mathrm{GA}$ indexed gene values. The GA gene classification methods with different Classifiers has shown a high accuracy than the existing SVM classifier.

\section{REFERENCES}

[1]. Mark A. Iwen, Willis Lang and Jignesh M. Patel, "Scalable Rule-Based Gene Expression Data Classification", In Proceedings of the IEEE 24th International Conference on Data Engineering, 2008

[2]. Nguyen and Rocke, Classification of Acute Leukemia based on DNA Micro array Gene Expressions using Partial Least Squares, Kluwer Academic, Dordrecht, 2001

[3]. Jian J. Dai, Linh Lieu, and David Rocke, "Dimension Reduction for Classification with Gene Expression Micro array Data", Statistical Applications in Genetics and Molecular Biology: Vol. 5, No. 1, 2006

[4]. Alok Sharma and Kuldip K. Paliwal, "Cancer classification by gradient LDA technique using micro array gene expression data", Data \& Knowledge Engineering, Vol. 66, pp. 338-347, 2008

[5]. Yuh-Jye Lee and Chia-Huang Chao, "A Data Mining Application to Leukemia Micro array Gene Expression Data Analysis", International Conference on Informatics, Cybernetics and Systems (ICICS), Kaohsiung, Taiwan, 2003

[6]. Donald Geman, Bahman Afsari, Aik Choon Tan and Daniel Q. Naiman, "Micro array Classification from Several Two-Gene Expression Comparisons", In Proceedings of ICMLA, pp.583-585, 2008

[7]. Chun-Hou Zheng, Bo Li, Lei Zhang and Hong-Qiang Wang, "Locally Linear Discriminant Embedding for Tumor Classification", In Proceedings of ICIC, pp.1093-1100, 2008

[8]. Cheng-San Yang, Li-Yeh Chuang, Chao-Hsuan Ke and Cheng-Hong Yang, "A hybrid Feature Selection Method for Micro array Classification", International Journal of Computer Science, Vol. 35, No. 3, 2008

[9]. James J. Chen and Chun-Houh Chen, "Micro array Gene Expression", Encyclopedia of Biopharmaceutical Statistics, 2nd Edition, Marcel Dekker, Inc., pp. 599-613, 2003 
[10]. Gad Getz, Erel Levine and Eytan Domany, "Coupled two-way clustering analysis of gene micro array data", In Proceedings of the National Academy of Sciences, Vol. 97, No. 22, pp. 12079-12084, 2000

[11]. Andrej Kastrin, "Item Response Theory Modeling for Micro array Gene Expression Data", Advances in methodology and statistics, Vol. 6, No. 1, pp. 51-67, 2009

[12]. Srinivas Mukkamala, Qingzhong Liu, Rajeev Veeraghattam and Andrew H. Sung, "Computational Intelligent Techniques for Tumor Classification (Using Micro array Gene Expression Data)", In Proceedings of World Congress on Lateral Computing, 2005

[13]. Fei Pana, Baoying Wanga, Xin Hub and William Perrizoa, "Comprehensive vertical sample-based KNN/LSVM classification for gene expression analysis", Journal of Biomedical Informatics, Vol. 37, pp. 240-248, 2004

[14]. Peng Qiu, Z. Jane Wang and Ray Liu, "Ensemble Dependence Model-based Cancer Classification using Gene Micro array Data", IEEE International Workshop on Genomic Signal Processing and Statistics (GENSIPS), 2005

[15]. Seeja and Shweta, "Microarray Data Classification Using Support Vector Machine", International Journal of Biometrics and Bioinformatics (IJBB), Vol. 5, No. 1, pp. 10-15, 2011

[16]. Manoj Kumar, "The Widely Used Diagnostics "DNA Microarray"-A Review", American Journal of Infectious Diseases, Vol. 5, No. 3, pp. 207-218, 2009

[17]. Danh V. Nguyen, David M. Rocke, "Tumor Classification by Partial Least Squares Using Micro array Gene Expression Data", Bioinformatics, Vol. 18, No. 1, pp. 39-50, 2002

[18]. Pengyi Yang and Zili Zhang, "An Embedded Two-Layer Feature Selection Approach for Microarray Data Analysis", IEEE Intelligent Informatics Bulletin, Vol.10, No.1, pp. 24-32, 2009

[19]. Yee Hwa Yang and Natalie P. Thorne, "Normalization for Two-color cDNA Microarray Data", Science and Statistics: A Festschrift for Terry Speed, Vol. 40, pp. 403-418, 2003

[20]. Ahmad M. Sarhan, "Cancer Classification Based on Micro array Gene Expression Data Using DCT and ANN", Journal of Theoretical and Applied Information Technology, Vol. 6, No. 2, pp. 208-216, 2009

[21]. Bharathi and Natarajan, "Cancer Classification of Bioinformatics data using ANOVA", International Journal of Computer Theory and Engineering, Vol. 2, No. 3, pp. 369-373, June 2010

[22]. Bo Li, Chun-Hou Zheng, De-Shuang Huang, Lei Zhang and Kyungsook Han, ""Gene expression data classification using locally linear discriminant embedding", Computers in Biology and Medicine, Vol. 40, pp. 802810,2010

[23]. Xiaosheng Wang and Osamu Gotoh, "A Robust Gene Selection Method for Micro array-based Cancer Classification", Journal of Cancer Informatics, Vol. 9, pp. 15-30, 2010

[24]. Mallika and Saravanan, "An SVM based Classification Method for Cancer Data using Minimum Micro array Gene Expressions", World Academy of Science, Engineering and Technology, Vol. 62, No. 99, pp. 543-547, 2010

[25]. Chhanda Ray, "Cancer Identification and Gene Classification using DNA Micro array Gene Expression Patterns", International Journal of Computer Science Issues, Vol. 8, Issue 2, pp. 155-160, March 2011

[26]. Seeja and Shweta, "Microarray Data Classification Using Support Vector Machine", International Journal of Biometrics and Bioinformatics (IJBB), Vol. 5, No. 1, pp. 10-15, 2011

[27]. http://en.wikipedia.org/wiki/Sensitivity_and_specificity

[28]. AnandaKumar and Punithavalli, "Efficient Cancer Classification using Fast Adaptive Neuro-Fuzzy Inference System (FANFIS) based on Statistical Techniques", International Journal of Advanced Computer ScienceandApplicationspp.132-1372011.

[29]. Heiss, Held, Estévez, Perez,Holzmann and Perez, "Classification of Sleep Stages in Infants: A Neuro Fuzzy Approach", IEEE Engineering in Medicine and Biology Magazine, Vol. 21, No. 5, pp. 147-51., 2002 